\title{
Microvascular Changes in Macular Area After Phacoemulsification and Its Influencing Factors Assessed by Optical Coherence Tomography Angiography
}

Jun Liu

Qiang Liu

Hua Yu

Ye Xia

Hui Zhang

Chao Geng

Lihong Dong

Department of Ophthalmology, Chaohu Hospital of Anhui Medical University, Hefei, 238000, Anhui, People's Republic of China
Correspondence: Lihong Dong Department of Ophthalmology, Chaohu Hospital of Anhui Medical University, Hefei, 238000, Anhui, People's Republic of China

Tel +86-55I-82324267

Email dlhyp6583@sina.com
Objective: To investigate macular vascular changes and underlying influencing factors using optical coherence tomography angiography (OCTA) after phacoemulsification in patients with cataracts.

Methods: Patients diagnosed with cataracts at the First Affiliated Hospital of Anhui Medical University from March to September 2019 were included. The macular retinal thickness, the microvascular density in the foveal, parafoveal, and perifoveal area, and the area of the foveal avascular zone (FAZ) were measured using OCTA at baseline, 1-week, 1-month, and 3-month post-operation.

Results: Forty-seven cataract patients (58 eyes) were eligible for this study. Compared with baseline thickness, the foveal, parafoveal, and perifoveal thicknesses, particularly in the inner retina, significantly increased at 1 - and 3-month post-operation $(\mathrm{P}<0.05)$. There was a nonsignificant difference in the microvascular density of the foveal and perifoveal areas at 1-week, 1-month, and 3-month post-operation $(\mathrm{P}>0.05)$. At 1 -month post-operation, the deep vessel density at the perifovea significantly increased $(\mathrm{P}<0.05)$. The FAZ area significantly diminished at 1 - and 3-month post-operation compared with the baseline data $(\mathrm{P}<0.05)$.

Conclusion: Phacoemulsification offers a satisfactory efficacy response, with an increased macular thickness, reduced FAZ area, and nonsignificant changes in the microvascular density at the perifovea after surgery.

Keywords: cataracts, phacoemulsification, optical coherence tomography, macular thickness, microvascular density

\section{Introduction}

Phacoemulsification with intraocular lens implantation for cataracts is one of the most common ophthalmic surgeries to improve the visual quality of cataract patients. ${ }^{1}$ Several studies have reported that changes in macular vascular density and blood flow areas after phacoemulsification may affect the recovery of visual acuity. $^{2}$ However, current studies mainly focus on the efficacy of phacoemulsification in pulsatile ocular blood flow changes or retinal macrovascular changes. The reaction of microvascular networks in response to the therapy is unclear, and there is still controversy about the effects of phacoemulsification on the macular thickness and microvascular changes in cataracts. ${ }^{2}$ 
Though recent studies have not provided sufficient evidence to figure out how the microvascular system changes in the macula after cataract surgery, most of them have suggested that damages to the blood-retinal barrier (BRB) are the most important cause of macular edema after surgery. ${ }^{3}$ Ferrari et $\mathrm{al}^{4}$ proposed that phacoemulsification energy might lead to macular edema after surgery. Fundus angiography revealed that greater energy from the surgery or longer operation time would pose a higher risk of BRB dysfunction in the operated eye, which are two popular indicators to measure the ultrasonic emulsification energy level at present. Due to dispersed energy distribution throughout the operative field and period, observation of the two single measurements cannot accurately reflect the ultrasonic energy level from the whole. The cumulative dissipated energy (CDE) is the average phacoemulsification power multiplied by total phacoemulsification time. This algorithm describes the ultrasonic energy level more accurately ${ }^{5}$ and aids in the monitoring of energy delivery to the macula intraoperatively.

Optical coherence tomography angiography (OCTA) is the latest microvascular imaging method widely used in retinal microvascular imaging. ${ }^{6}$ OCTA using the frequency division based decorrelation algorithm can yield retinal three-dimensional (3D) microvascular images. This noninvasive, high-resolution technique is superior in exemption from contrast agent injection, high-speed imaging to capture blood flow, and 3D imaging of microvascular structures at different depths, compared with traditional angiographic techniques. So it has wider applicability.

In this work, we investigated changes in macular retinal thickness and microvascular blood flow using OCTA after phacoemulsification in cataract patients and modulating factors, aiming to explore the underlying mechanism of microvascular changes affecting the visual quality of patients after phacoemulsification.

\section{Materials and Methods General Data}

A total of 50 patients (61 eyes), including 25 males (29 eyes) and 25 females ( 32 eyes), who underwent phacoemulsification at the First Affiliated Hospital of Anhui Medical University from March to September 2019 were included. If a patient required cataract surgery in both eyes, only the operated eye was included. Patients were eligible for this study if they (1) had an intraocular pressure $\leq 21 \mathrm{mmHg}(1 \mathrm{kPa}=7.5 \mathrm{mmHg})$ in the affected eye, (2) showed the absence of severe cataracts or unstable head fixation affecting OCTA signal acquisition, and (3) reported no history of other ocular diseases (such as uveal tract, retina, glaucoma), systemic diseases like diabetes, and previous intraocular surgery. All patients agreed to participate in the study and signed an informed consent form. The permission of this study was conformed to the principles of the Declaration of Helsinki. Preoperative eye examinations consisted of an assessment of visual function, slit lamp examination, trifacetoscopy, non-contact intraocular pressure measurement, as well as axial length and other measurements from the IOLMaster. The nuclear hardness was graded (grades 1-4) in terms of nuclear color and lens opacity using the Emery-Little classification system. The phacoemulsification time and energy of each patient were recorded throughout the surgery, and macular retinal thickness and microvascular blood flow were examined using OCTA before surgery and at 1-week, 1-month, and 3-month post-operation. All measurements were performed at 9:00-12:00 am to minimize the influence of diurnal variation. Fifty patients (61 eyes) completed the follow-up examination at 1 week, 49 cases (60 eyes) at 1 month, and 47 cases (58 eyes) at 3 months. Therefore, 47 patients (58 eyes) were eligible for this study. In accordance with the principles of the Declaration of Helsinki, all subjects provided informed written consent prior to participation. The Ethics Committee of Chaohu Hospital of Anhui Medical University approved this study.

\section{Methods}

\section{Surgical Procedures}

Phacoemulsification was performed using the Alcon-Infiniti Vision System (Irish Health, USA). After the topical anesthetic $20 \mathrm{~g} \cdot \mathrm{L}^{-1}$ lidocaine was instilled into the eye, a $2.2 \mathrm{~mm}$ clear corneal self-sealing incision was made. Once the posterior capsule tear appeared, pieces of lens nucleus were extracted, and the residual lens cortex was removed using phacoemulsification. After lens materials were completely removed, sodium hyaluronate was injected into the anterior chamber, and a foldable intraocular lens was implanted in the capsular bag. Postoperatively, tobramycin dexamethasone and $5 \mathrm{~g} \cdot \mathrm{L}^{-1}$ levofloxacin eye drops were given four times a day for 2 weeks, and pranoprofen eye drops four times a day for 4 weeks.

\section{OCTA Imaging}

OCTA was performed using RTvue-XR Avanti OCT (Optovue RTVue XR Avanti; Optovue, Inc., Fremont, CA, USA) that ran at a speed of 70,000 A-scans per second. The split-spectrum amplitude-decorrelation angiography (SSADA) algorithm was implemented to enhance visualization of the macular 
vasculature and obtain OCTA images $(304 \times 304$ pixels). The OCTA system utilized a light source centered on a wavelength of $840 \mathrm{~nm}$ and a bandwidth of $50 \mathrm{~nm}$. Patients with the repeatability of OCTA parameters $>40$ could be included in our study. OCT scans were performed from the inner limiting membrane (ILM) to the retinal pigment epithelium (RPE). The fovea $(\varnothing=1 \mathrm{~mm})$, parafovea $(\varnothing=3 \mathrm{~mm})$, and perifovea $(\varnothing=$ $6 \mathrm{~mm}$ ) were three circular areas.

The macular retinal thickness referred to the average thickness of the macula, and the entire retinal thickness (full thickness) was the distance from the ILM to the RPEBruch's membrane complex. The retina between the ILM and the outer boundary of the inner plexiform layer (IPL) was the inner retina, and that between the outer boundary of the IPL and the RPE-Bruch's membrane complex the outer retina. The OCTA system could automatically yield the three average thicknesses: full, inner retinal layer, and outer retinal layer thicknesses (FT, IRLT, ORLT).

As for the determination of macular retinal microvascular density, OCTA images of the deep and superficial retinal layers were used for analysis in this study. The superficial retina was defined as $3 \mu \mathrm{m}$ below the internal limiting membrane (ILM) to $15 \mu \mathrm{m}$ below the inner plexiform layer (IPL), and the deep retina as 15-70 $\mu \mathrm{m}$ below the IPL. The macular blood flow and the area of the foveal avascular zone (FAZ) were analyzed using OCTA images and Image J software.

All scans were performed by one experienced physician (zhanghui) to ensure the consistency and accuracy of OCTA data. It has been reported that lens opacities have a significant influence on retinal blood flow measurements in OCTAand should be considered in quantitative vessel analysis. ${ }^{7}$ Thus, in order to avoid the influence of lens opacity on evaluation of macular structure using OCTA, the signal values of OCTA of each patient were all above Q5.

And signal values of OCTA of each patient were above Q5. Patients were allowed to undertake examinations at 9:0012:00 am under constant room lighting to eliminate the influence of diurnal variation and light intensity on macular thickness.

\section{Statistical Analysis}

SPSS 17.0 statistical software was used for statistical analysis. All data were expressed as means \pm standard deviation (SD). Baseline and postoperative data were compared using repeated-measures analysis of variance, followed by Fisher's LSD test for pairwise comparison. The Chi-square and $t$-test were used for comparisons between different groups. A $p$-value $<0.05$ was considered statistically significant.

\section{Results}

\section{Clinical and Demographic Characteristics}

Of the 58 eyes (47 patients, average age: $66.26 \pm 11.92$ years, range: $32-85$ years), the preoperative best-corrected visual acuity (BCVA) was $0.25 \pm 0.14(0.02-0.60)$, the axial length was $23.79 \pm 1.40(21.32-28.42) \mathrm{mm}$, the mean nuclear hardness grade was $2.26 \pm 0.54$ (grades $1-4$ ), the effective phacoemulsification time (EPT) was $0.93 \pm 0.55$ $(0.14-2.97) \mathrm{s}$, and the mean phacoemulsification energy was $22.65 \% \pm 6.97 \%(7.50-32.80 \%)$. The eyes were stratified into two energy level groups, with the first 29 eyes into the low-energy group and the last 29 into the highenergy group. The CDE (phacoemulsification energy $x$ phacoemulsification time) of the low-energy group was $0.11 \pm 0.06$, significantly lower than that of $0.32 \pm 0.12$ in the high-energy group $(\mathrm{P}<0.05)$. Besides, the intraocular pressure of all patients at 1- and 3-months postoperation $(14.53 \pm 3.30$ and $13.74 \pm 2.86 \mathrm{mmHg})$ significantly decreased compared with the baseline level (15.86 \pm $3.67 \mathrm{mmHg}$ ) (both $\mathrm{P}<0.05$ ).

\section{Comparisons of Nuclear Hardness and Postoperative BCVA Between Low- versus High-Energy Groups}

The number of eyes with grade 3 nuclear hardness in the high-energy group was significantly higher than that in the low-energy group $(\mathrm{P}<0.05)$. The postoperative BCVA of the low-energy group was higher than that of the highenergy group at 1 -week $(0.83 \pm 0.17$ vs $0.70 \pm 0.16, \mathrm{P}<$ $0.05)$ and 1 -month $(0.86 \pm 0.14$ vs $0.77 \pm 0.16, \mathrm{P}<0.05)$ post-operation. However, there was a nonsignificant difference in postoperative BCVA between the two groups at 3 months $(\mathrm{P}>0.05)$, as revealed in Table 1 .

\section{Changes in Foveal, Parafoveal, and Perifoveal Retinal Thickness Before and After Surgery}

Compared with preoperative values, the FTs at the fovea, parafovea, and perifovea significantly increased at 1- and 3 -month post-operation ( $\mathrm{P}<0.05$ ) (Table 2). Representative images of the macular retinal thickness were shown in Figure 1. The IRLTs increased more tremendously at the parafovea and perifovea at 1 - and 3-month post-operation ( $\mathrm{P}$ $<0.01$ ), and changes in ORLTs were nonsignificant except for a mild decrease in the perifoveal region. 
Table I Comparisons of Nuclear Hardness and Postoperative BCVA Between Low- and High-Energy Groups

\begin{tabular}{|c|c|c|c|c|}
\hline & $\begin{array}{l}\text { Low-Energy Group } \\
\qquad(n=29)\end{array}$ & $\begin{array}{l}\text { High-Energy Group } \\
\qquad(n=29)\end{array}$ & $\chi^{2} / t$ & $P$ \\
\hline \multicolumn{5}{|l|}{ Lens grade } \\
\hline Grade 2 & 19 & 6 & 11.560 & 0.003 \\
\hline Grade 3 & 8 & 19 & & \\
\hline Grade 4 & I & 2 & & \\
\hline \multicolumn{5}{|l|}{ BCVA } \\
\hline Baseline & $0.29 \pm 0.13$ & $0.27 \pm 0.10$ & 0.638 & 0.526 \\
\hline I-week post-operation & $0.83 \pm 0.17$ & $0.70 \pm 0.16$ & 2.951 & 0.005 \\
\hline I-month post-operation & $0.86 \pm 0.14$ & $0.77 \pm 0.16$ & 2.226 & 0.030 \\
\hline 3-month post-operation & $0.88 \pm 0.13$ & $0.81 \pm 0.14$ & 1.975 & 0.053 \\
\hline
\end{tabular}

Table 2 Changes in Foveal, Parafoveal, and Perifoveal Retinal Thickness Before and After Surgery

\begin{tabular}{|c|c|c|c|c|c|}
\hline & \multicolumn{4}{|c|}{ 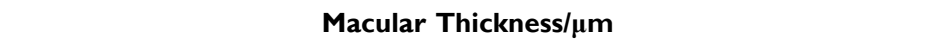 } & \multirow[t]{2}{*}{$P$} \\
\hline & Baseline & I Week & I Month & 3 Months & \\
\hline \multicolumn{6}{|l|}{ Full thickness } \\
\hline Fovea & $265.5 \pm 52.5$ & $261.3 \pm 28.6$ & $275.3 \pm 46.7 * *$ & $275.6 \pm 31.5^{* *}$ & 0.05 \\
\hline Parafovea & $321.4 \pm 26.0$ & $325.8 \pm 18.2$ & $334.4 \pm 23.0 * *$ & $335.7 \pm 19.9 * *$ & $<0.01$ \\
\hline Perifovea & $286.3 \pm 23.5$ & $285.3 \pm 16.9$ & $292.7 \pm 18.2^{*}$ & $294.1 \pm 19.8^{*}$ & 0.001 \\
\hline \multicolumn{6}{|c|}{ Outer retinal layer thickness } \\
\hline Fovea & $223.4 \pm 57.4$ & $204.8 \pm 29.4$ & $220.7 \pm 37.0$ & $219.0 \pm 22.9$ & 0.073 \\
\hline Parafovea & $237.8 \pm 40.8$ & $223.6 \pm 23.6$ & $225.3 \pm 19.8$ & $226.3 \pm 20.2$ & 0.064 \\
\hline Perifovea & $196.4 \pm 23.6$ & $184.2 \pm 13.0 * *$ & $186.5 \pm 10.0^{*}$ & $189.4 \pm \mid 2.2$ & 0.001 \\
\hline \multicolumn{6}{|c|}{ Inner retinal layer thickness } \\
\hline Fovea & $50.0 \pm 36.4$ & $53.4 \pm 14.3$ & $55.5 \pm 14.3$ & $60.3 \pm 26.6$ & 0.292 \\
\hline Parafovea & $84.8 \pm 25.2$ & $103.4 \pm 17.7^{* *}$ & $109.3 \pm 13.2^{* *}$ & $\mid I 4.1 \pm 24.9 * *$ & $<0.01$ \\
\hline Perifovea & $92.3 \pm 10.2$ & $102.6 \pm 12.1 * *$ & $107.7 \pm 11.2 * *$ & $108.9 \pm 19.8^{* *}$ & $<0.01$ \\
\hline
\end{tabular}

Notes: $* P<0.05, * * P<0.01$, versus baseline.

\section{Changes in Macular Retinal Microvascular} Density at the Parafovea and Perifovea

Compared with baseline levels, there were no significant changes in the superficial microvascular density in the parafoveal and perifoveal regions at 1-week, 1-month, and 3-month post-operation $(\mathrm{P}>0.05)$. Representative image of the superficial microvascular density were shown in Figure 2. The deep microvascular density in the parafoveal and perifoveal regions merely showed a slightly increasing trend, but the deep microvascular density at the fovea significantly increased at 1 month $(\mathrm{P}<0.05)$, as shown in Table 3.

\section{Changes in the FAZ Area Before and After Cataract Surgery}

The FAZ area at 1-month post-operation was $0.42 \pm$ $0.23 \mathrm{~mm}^{2}$, significantly smaller than the preoperative value of $0.73 \pm 0.91 \mathrm{~mm}^{2}(\mathrm{P}<0.05)$. The FAZ area at 3 months $\left(0.34 \pm 0.17 \mathrm{~mm}^{2}\right)$ also pronouncedly decreased compared 

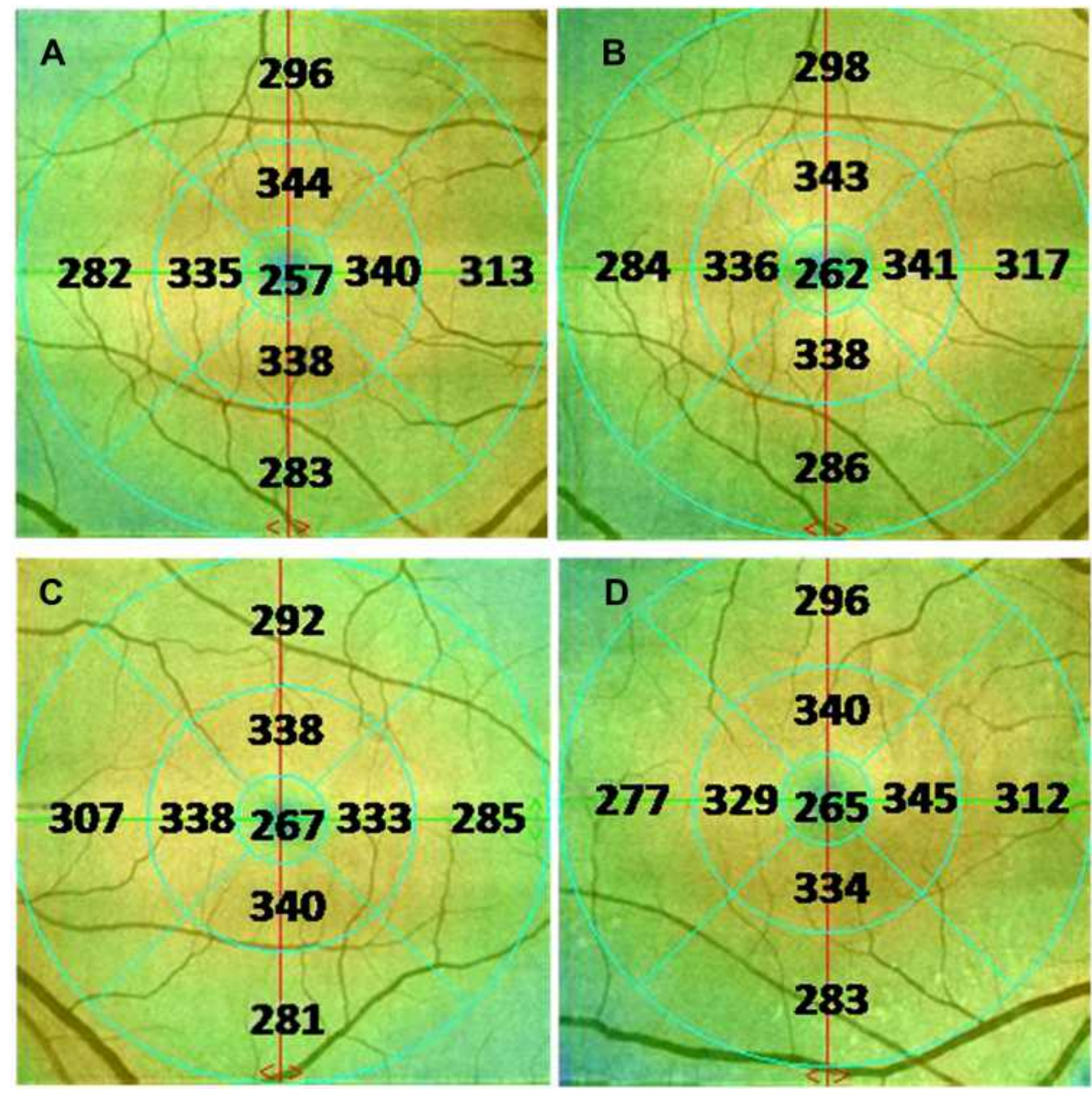

Figure I Representative images of the macular retinal thickness. (A) Baseline; (B) I-week; (C) I-month; (D) 3-month.

with the baseline level $(\mathrm{P}<0.05)$. Representative images of the FAZ area were shown in Figure 3.

\section{Comparisons of Foveal Retinal Thicknesses Between Low- versus High-Energy Groups}

There were no significant differences in foveal retinal thicknesses in terms of FT, IRLT, and ORLT between the low- and high-energy groups at baseline $(\mathrm{P}>0.05)$. Neither were variations between them postoperatively ( $\mathrm{P}$ $>0.05$ ), as shown in Table 4 .

\section{Comparisons of Microvascular Blood Flow in the Central Fovea Between Low- versus High-Energy Groups}

There was a nonsignificant difference in the microvascular blood flow in the central fovea between the low- and highenergy groups preoperatively $(\mathrm{P}>0.05)$. Neither were changes between them at the three follow-up time points ( $\mathrm{P}$ $>0.05$ ), as shown in Table 5 .

\section{Comparison of the FAZ Area Between Low- versus High-Energy Groups}

Also, there were nonsignificant differences in the FAZ area between the low- and high-energy groups at baseline and the three follow-up time points $(\mathrm{P}>0.05)$, as shown in Table 6. However, there were decreases in the FAZ area after surgery in each group.

\section{Discussion}

This study demonstrates changes in macular retinal thickness and vasculature using OCTA after phacoemulsification. There were remarkable increases in retinal thicknesses in the macular area, especially at the inner retina, and the deep microvascular density in the parafoveal and perifoveal regions tended to increase nonsignificantly after surgery. 

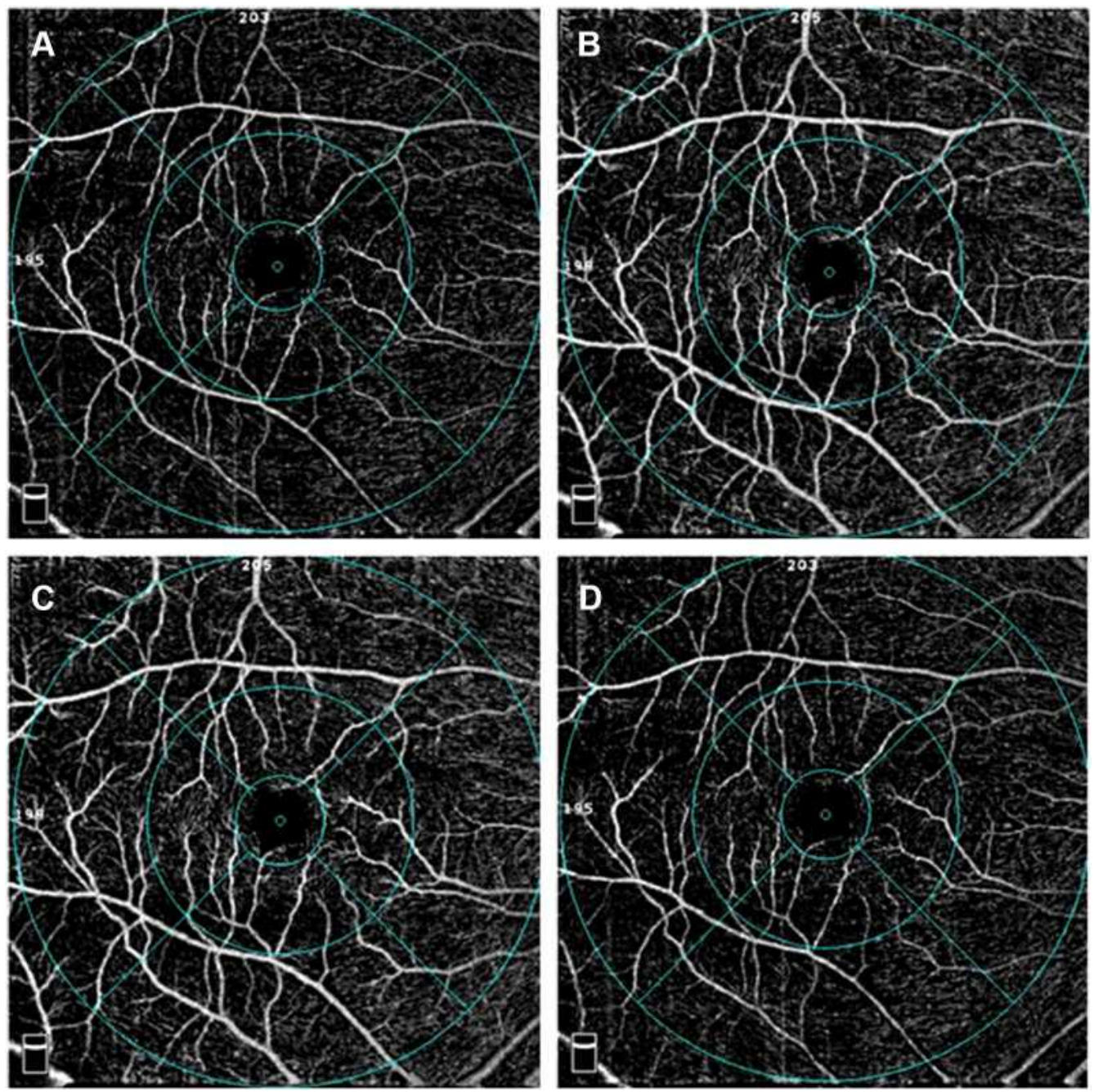

Figure 2 Representative images of the superficial microvascular density. (A) Baseline; (B) I-week; (C) I-month; (D) 3-month.

Besides, there were decreases in the FAZ area after surgery. The underlying effect of influencing factor, ultrasound energy from phacoemulsification, on the macula was explored by stratifying patients into the low- and high- energy groups. The postoperative BCVA in the high-energy group was seemingly not as good as that in the low-energy group but without a significant difference between the two groups at 3-month post-operation. No significant differences

Table 3 Changes in the Macular Retinal Microvascular Density at the Parafovea and Perifovea

\begin{tabular}{|l|l|l|l|l|l|}
\hline \multirow{2}{*}{} & \multicolumn{3}{|c|}{ Macular Retinal Microvascular Density/\% } & \multicolumn{1}{c|}{ P } \\
\cline { 2 - 6 } & Baseline & I Week & I Month & 3 Months \\
\hline Superficial microvascular density & & & & & \\
$\quad$ Parafovea & $43.3 \pm 7.8$ & $41.3 \pm 6.2$ & $42.8 \pm 5.6$ & $42.2 \pm 5.7$ & 0.643 \\
$\quad$ Perifovea & $47.2 \pm 8.6$ & $45.3 \pm 5.6$ & $46.0 \pm 4.8$ & $45.4 \pm 4.6$ & 0.565 \\
\hline $\begin{array}{l}\text { Deep microvascular density } \\
\text { Parafovea }\end{array}$ & & & & & \\
Perifovea & $43.2 \pm 13.2$ & $45.5 \pm 9.9$ & $48.9 \pm 8.2$ & $46.4 \pm 9.6$ & 0.130 \\
\hline
\end{tabular}

Notes: $* * P<0.01$, versus baseline. 

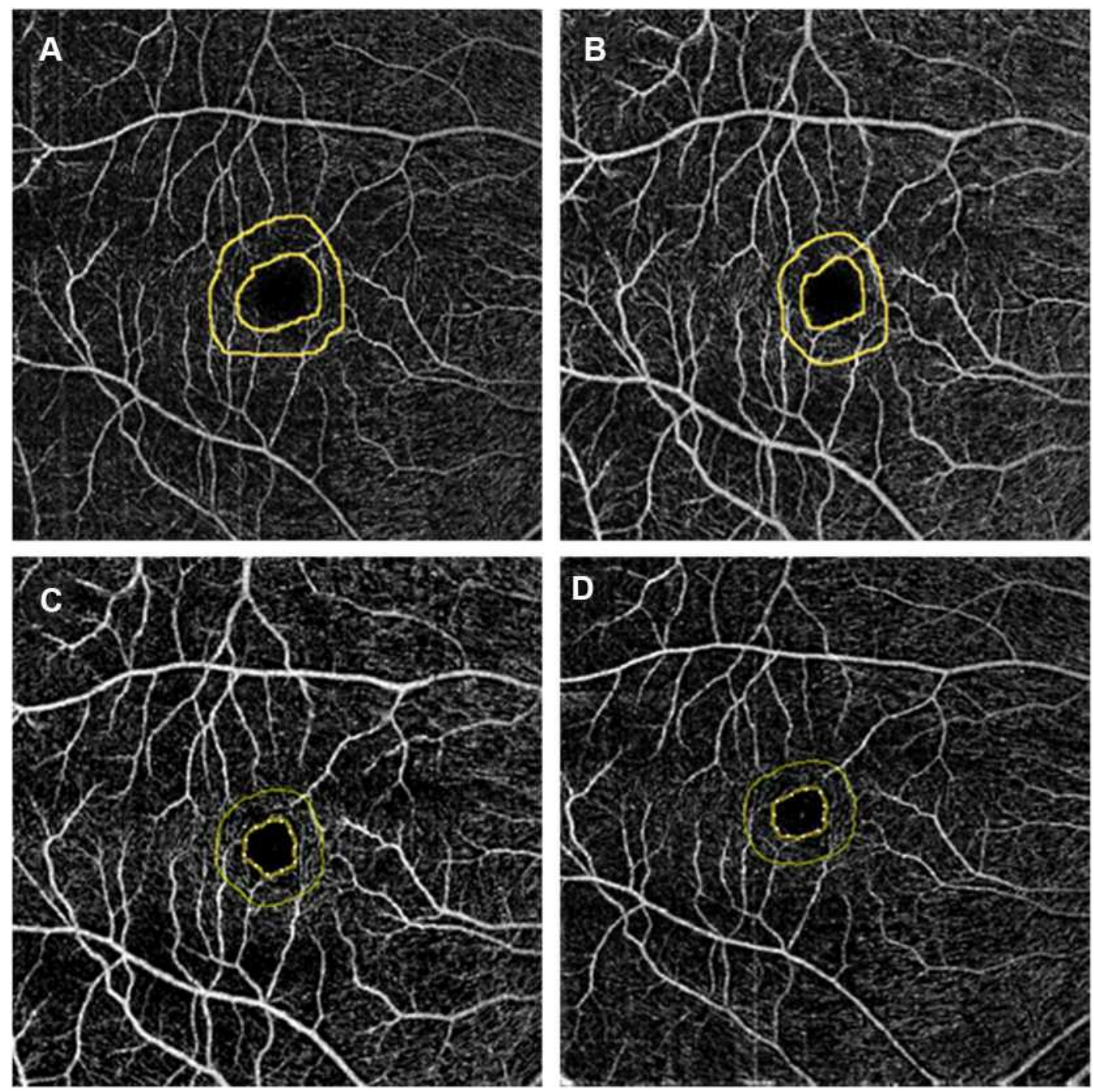

Figure 3 Representative images of the FAZ area. (A) Baseline; (B) I-week; (C) I-month; (D) 3-month.

were found in postoperative foveal retinal thicknesses and microvascular blood flow between the two energy level groups.

Macular edema is one of the main complications after phacoemulsification for cataracts considered to have an impact on the recovery of postoperative visual function in cataract patients. It is mainly manifested in changes in the foveal retinal thickness and macular microvasculature after surgery. ${ }^{8}$ In this study, markedly increased IRLT after phacoemulsification may delay the recovery of the visual acuity in patients. There is no ascertainment on the mechanism upon which phacoemulsification leads to increased retinal thicknesses in the macula. Some studies have suggested that an increase in the macular retinal thickness may result from BRB damages. ${ }^{3}$ Vitreous detachment that occurs during surgery can result in vitreomacular traction, which further damages the BRB more severely. ${ }^{9}$ Excessive release of inflammatory factors, on the other hand, is also a potential cause of BRB disruption. Evidence shows significant increases in pro-inflammatory proteins such as chemokine 2 and interleukin- $1 \beta$ in the neurosensory retina of mice following lens extraction, which are believed to cause vasodilatation, contributing to BRB disruption. ${ }^{10}$ Other studies reported that increased permeability of capillaries in the macula and decreased barrier function of retinal pigment epithelial cells might also lead to macular retinal edema, featuring increased retinal thickness. ${ }^{11}$ Therefore, how to minimize inflammatory responses at the macula intraoperatively to avoid vitreomacular traction and mitigate macular edema are worthy of further study and discussion for a speedy recovery of visual acuity in cataract patients after surgery. 
Table 4 Comparison of Foveal Retinal Thickness Between Low- versus High-Energy Groups

\begin{tabular}{|c|c|c|c|c|}
\hline & Low-Energy Group $(n=29)$ & High-Energy Group $(n=29)$ & $\chi^{2} / t$ & $\boldsymbol{P}$ \\
\hline \multicolumn{5}{|l|}{ Inner retinal layer thickness } \\
\hline Baseline & $45.29 \pm 14.74$ & $50.52 \pm 44.56$ & 0.589 & 0.558 \\
\hline I-week post-operation & $54.04 \pm \mid 1.27$ & $52.67 \pm 18.13$ & 0.338 & 0.737 \\
\hline I-month post-operation & $53.29 \pm 18.67$ & $54.63 \pm|5.7|$ & 0.288 & 0.774 \\
\hline 3-month post-operation & $49.25 \pm|2.6|$ & $55.78 \pm 11.59$ & 1.997 & 0.051 \\
\hline \multicolumn{5}{|l|}{ Outer retinal layer thickness } \\
\hline Baseline & $2|4.2| \pm 33.37$ & $226.15 \pm 40.20$ & 1.200 & 0.236 \\
\hline I-week post-operation & $217.68 \pm 57.94$ & $200.96 \pm 39.30$ & 1.248 & 0.218 \\
\hline I-month post-operation & $2|4.79 \pm 58.1|$ & $223.30 \pm 37.00$ & 0.645 & 0.522 \\
\hline 3-month post-operation & $220.61 \pm 23.16$ & $212.96 \pm 25.22$ & I.I7I & 0.247 \\
\hline \multicolumn{5}{|l|}{ Full thickness } \\
\hline Baseline & $260.57 \pm 31.4 I$ & $268.19 \pm 36.72$ & 0.827 & 0.412 \\
\hline I-week post-operation & $261.00 \pm 24.07$ & $257.22 \pm 35.77$ & 0.461 & 0.647 \\
\hline I-month post-operation & $268.43 \pm 72.69$ & $270.15 \pm 42.31$ & 0.107 & 0.915 \\
\hline 3-month post-operation & $270.68 \pm 24.98$ & $275.89 \pm 34.76$ & 0.640 & 0.525 \\
\hline
\end{tabular}

Table 5 Comparisons of Microvascular Blood Flow in the Central Fovea Between Low- versus High-Energy Groups

\begin{tabular}{|l|c|c|c|c|}
\hline & Low-Energy Group (n=29) & High-Energy Group (n=29) & $\chi^{2} / \mathbf{t}$ & \\
\hline Superficial layers & & & 0.276 & 0.784 \\
Baseline & $10.01 \pm 7.87$ & $9.29 \pm 11.29$ & 1.823 & 0.074 \\
I-week post-operation & $15.02 \pm 9.03$ & $11.06 \pm 6.92$ & 1.396 & 0.168 \\
I-month post-operation & $14.92 \pm 9.65$ & $11.62 \pm 7.71$ & 1.178 & 0.224 \\
3-month post-operation & $15.99 \pm 10.53$ & $12.69 \pm 10.26$ & 0.632 & \\
\hline Deep layers & & & 1.823 & 0.530 \\
Baseline & $21.41 \pm 13.92$ & $18.94 \pm 14.96$ & 1.396 & 0.074 \\
I-week post-operation & $15.02 \pm 9.03$ & $11.06 \pm 6.92$ & 1.178 & 0.168 \\
I-month post-operation & $14.92 \pm 9.65$ & $11.62 \pm 7.71$ & $12.69 \pm 10.26$ & 0.244 \\
3-month post-operation & $15.99 \pm 10.53$ & & \\
\hline
\end{tabular}

Table 6 Comparison of the FAZ Area Between Low- versus High-Energy Groups

\begin{tabular}{|l|c|c|c|c|}
\hline & Low-Energy Group (n=29) & High-Energy Group (n=29) & $\chi^{\mathbf{2} / \mathbf{t}}$ & $\boldsymbol{P}$ \\
\hline Baseline & $0.49 \pm 0.57$ & $0.79 \pm 0.83$ & 1.557 & 0.125 \\
I-week post-operation & $0.38 \pm 0.41$ & $0.45 \pm 0.21$ & 0.760 & 0.450 \\
I-month post-operation & $0.36 \pm 0.20$ & $0.44 \pm 0.21$ & 1.356 & 0.181 \\
3-month post-operation & $0.33 \pm 0.16$ & $0.39 \pm 0.26$ & 1.171 & 0.247 \\
\hline
\end{tabular}

Our results merely revealed slightly increasing trends in the deep microvascular density at the parafovea and perifovea, as well as enhanced pulsatile ocular blood flow after phacoemulsification, which might attribute to the decreased mean intraocular pressure. ${ }^{2}$ Other studies reported that the fundus pulsation amplitude decreases with intraocular pressure increases. ${ }^{12}$ Therefore, our finding that the intraocular pressure at 1- and 3-month postoperation was significantly lower than the baseline levels can partly explain the vascular changes in the macular 
area. Hilton et $\mathrm{al}^{2}$ reported that increased ocular pulsatile blood flow after cataract surgery was beneficial; however, whether this aids in a slower progression of diabetic retinopathy is unclear. Another possible cause of macular vascular changes is an increase in the amount of light after cataract surgery, as supported by pieces of evidence that cataracts may block $18 \%$ to $40 \%$ of light at different wavelengths. ${ }^{13}$ Increased light exposure may also have some association with the relatively high incidence of postoperative macular degeneration. ${ }^{14}$ Whether increased light can also lead to more activity and more metabolic demand in the retina, which in turn leads to an increase in blood flow, is unclear and needs to be demonstrated by further studies.

This study also reported a decreasing trend in the FAZ area. The FAZ is an avascular area in the center of the fovea surrounded by a capillary ring. The FAZ area is crucial to the central visual function, and once affected, it may result in varying degrees of visual loss. ${ }^{15}$ Studies have shown that a larger FAZ area is associated with the severity of capillary occlusion and nonperfusion in the macular region of the retina. ${ }^{16} \mathrm{~A}$ recent study reported a negative correlation between the degree of FAZ enlargement and visual acuity loss in patients with diabetic retinopathy, and similarly, there was also a decreasing trend in the FAZ area after surgery. ${ }^{17}$ Zhao et $\mathrm{al}^{18}$ reported that the FAZ area decreased by $27 \%$ three months after phacoemulsification, and there might link to the good recovery of visual acuity. Consistently, our study found remarkable decreases in the FAZ area at 1 and 3 months after phacoemulsification, which may explain the improved visual quality of cataract patients. The exact reason for the decrease in FAZ is not yet clear. Two explanations should be suggested: (1) Reduction of retinal edema. Retinal edema can promote angiogenesis to the center and reduce the area of FAZ. Thus, the reduction of retinal edema may be one of the reasons for the decrease in FAZ. (2) Change in refractive power. The myopia of the dense eyes of cataract patients can shift up to 8 diopters, which may "falsely" increase the area of FAZ. After phacoemulsification, the eye becomes emmetropic and the area of FAZ may be decreased. Further researches are needed to confirm these assumptions.

Besides, the visual acuity of patients in the high-energy group was not as good as that in the low-energy group at 1-week and 1-month post-operation, notwithstanding a nonsignificant difference in the visual acuity between the two groups at 2 months. This may attribute to longer operation time alongside greater energy from phacoemulsification in the high-energy group, which may lead to postoperative corneal edema, incision astigmatism, aqueous flare, and macular edema and consequently hinder a speedy recovery postoperatively. In terms of the macular retinal thickness and blood flow, no significant differences were found between the low- and high-energy groups at 1-week, 1-month, and 2-month post-operation $(\mathrm{P}>0.05)$. Consistently, Sourdille et $\mathrm{al}^{19}$ and Cheng et $\mathrm{al}^{20}$ found that more severe retinal injury was related to stronger ultrasonic energy during phacoemulsification. Wang et $\mathrm{al}^{5}$ performed a correlation analysis and found that higher energy accumulation promoted nuclear hardness of the lens. However, there was no significant difference between the two groups in this study. Therefore, considering that the majority of cataract nucleus hardness in this study is grade II and III, the phacoemulsification energy and phacoemulsification time used during the operation are relatively small. Even in the high cumulative energy group, The cumulative dissipated energy (CDE) is $(0.32 \pm 0.12)$, which showed a nonsignificant difference in macular retinal blood flow between the low- and high-energy groups. This finding suggests that during phacoemulsification, the incidence of macular edema can be lowered by minimizing the operation time and reducing ultrasound energy to diminish the pruritus and damage of intraocular tissues.

This study had some limitations. First, this study is a single-center study and only used data from a single hospital. Thus, a selection bias may have been introduced. Second, the sample size is relatively small, and the postoperative observation period was relatively short. Therefore, larger multi-center studies with larger sample sizes are recommended to confirm these results.

Phacoemulsification followed by intraocular lens implantation offered amelioration, including increased macular retinal thickness and decreased FAZ area, without major variations in the microvascular density. Macular edema induced by intraoperative energy accumulation from phacoemulsification might hinder the postoperative recovery of visual acuity within 1 month, and this effect was nonsignificant at 3 months. The conclusion of our study needs further validations by large-sample studies. 


\section{Disclosure}

The authors report no conflicts of interest in this work.

\section{References}

1. Chen Z, Song F, Sun L, et al. Corneal integrity and thickness of central fovea after phacoemulsification surgery in diabetic and nondiabetic cataract patients. Arch Med Sci. 2018;14:818-825. doi:10.5114/ aoms.2016.64036

2. Hilton EJ, Hosking SL, Gherghel D, et al. Beneficial effects of small-incision cataract surgery in patients demonstrating reduced ocular blood flow characteristics. Eye. 2004;19:670-675. doi:10.1038/sj. eye. 6701620

3. Hussein KA, Choksi K, Akeel S, et al. Bone morphogenetic protein 2: a potential new player in the pathogenesis of diabetic retinopathy. Exp Eye Res. 2014;125:79-88. doi:10.1016/j.exer.2014.05.012

4. Ferrari TM, Cavallo M, Durante G, et al. Macular edema included by phacoemulsification. Doc Ophthalmol. 1999;97(3/4):325-327. doi:10.1023/a:1002142307952

5. Wang XQ, Zhou IY, Huang YL. Clinical significance of accumulated energy complex parameter in phacoemulsification. Zhonghua Yan Ke Za Zhi. 2002;38:610-613.

6. Cohen SY, Miere A, Nghiem-Buffet S, et al. Clinical applications of optical coherence tomography angiography: what we have learnt in the first 3 years. Eur J Ophthalmol. 2018;28(5):491-502. doi:10.1177/ 1120672117753704

7. Yu S, Frueh BE, Steinmair D, et al. Cataract significantly influences quantitative measurements on swept-source optical coherence tomography angiography imaging. PLoS One. 2018;13(10):e0204501. doi:10.1371/journal.pone.0204501

8. Desapriya E, Subzwari S, Scime-Beltrano G, et al. Vision improvement and reduction in falls after expedited cataract surgery systematic review and metaanalysis. J Cataract Refract Surg. 2010;36(1):13-19. doi:10.1016/j.jcrs.2009.07.032

9. Cong WX, Zhang ZC, Hao XL. Mechanism of the macular fovea thickness change after cataract surgery in diabetic patients. Int Eye Sci. 2017;17:888-890. doi:10.3980/j.issn.1672-5123.2017.5.21
10. Xu H, Chen M, Forrester JV, et al. Cataract surgery induces retinal pro-inflammatory gene expression and protein secretion. Invest Ophthalmol Vis Sci. 2011;52:249-255. doi:10.1167/iovs.10-6001

11. Cheng GJ, Lin Y. Diabetic macular edema: pathogenesis and treatment. Surv Ophthalmol. 2009;54(1):1-32. doi:10.1016/j. survophthal.2008.10.001

12. Weigert G, Findl O, Luksch A, et al. Effects of moderate changes in intraocular pressure on ocular hemodynamics in patients with primary open-angle glaucoma and healthy controls. Ophthalmology. 2005;112:1337-1342. doi:10.1016/j.ophtha.2005.03.016

13. Artigas JM, Felipe A, Navea A, et al. Spectral transmission of the human crystalline lens in adult and elderly persons: color and total transmission of visible light. Invest Ophthalmol Vis Sci. 2012;53 (7):4076-4084. doi:10.1167/iovs.12-9471

14. Libre PE. Intraoperative light toxicity: a possible explanation for the association between cataract surgery and age-related macular degeneration. Am J Ophthalmol. 2003;136(5):961. doi:10.1016/ s0002-9394(03)00906-1

15. Wang X, Jia Y, Spain R, et al. Optical coherence tomography angiography of optic nerve head and parafovea in multiple sclerosis. $\mathrm{Br}$ $J$ Ophthalmol. 2014;98(10):1368-1373. doi:10.1136/bjophthalmol2013-304547

16. Matsunaga DR, Yi JJ, De Koo LO, et al. Optical coherence tomography angiography of diabetic retinopathy in human subjects. Ophthalmic Surg Lasers Imaging Retina. 2015;46(8):796-805. doi:10.3928/23258160-20150909-03

17. Zheng D, Pang DB. Evaluation of early macular retinal microcirculation in diabetic patients using optical coherence tomography angiography. Rec Adv Ophthalmol. 2018;38:548-552.

18. Zhao Z, Wen W, Jiang C, et al. Changes in macular vasculature after uncomplicated phacoemulsification surgery: optical coherence tomography angiography study. $J$ Cataract Refract Surg. 2018;44 (4):453-458. doi:10.1016/j.jcrs.2018.02.014

19. Sourlille P, Santiago PY. Optical coherence tomography of macular thickness after cataract surgery. $J$ Cataract Refract Surg. 1999;25:256-261. doi:10.1016/s0886-3350(99)80136-9

20. Cheng B, Liu YZ, Liu X, et al. Observation the changes of the macula after phacoemulsification with optical coherence tomography. Chin J Ocul Fundus Dis. 2002;38:265-267.
Therapeutics and Clinical Risk Management

\section{Publish your work in this journal}

Therapeutics and Clinical Risk Management is an international, peerreviewed journal of clinical therapeutics and risk management, focusing on concise rapid reporting of clinical studies in all therapeutic areas, outcomes, safety, and programs for the effective, safe, and sustained use of medicines. This journal is indexed on PubMed Central, CAS,

\section{Dovepress}

EMBase, Scopus and the Elsevier Bibliographic databases. The manuscript management system is completely online and includes a very quick and fair peer-review system, which is all easy to use. Visit http://www.dovepress.com/testimonials.php to read real quotes from published authors. 